\title{
Mothers' knowledge regarding peripheral intravenous catheter caring and complications among pediatric patients: A cross-sectional study
}

\author{
Rania Jaber, Huda Shaweesh, Alaa A. Zarqa, Othman A. Alfuqaha* \\ Department of Nursing, Jordan University Hospital, The University of Jordan, Amman, Jordan
}

Received: June 21, 2021

DOI: $10.5430 /$ jnep.v11n12p31

\author{
Accepted: July 22, 2021 \\ Online Published: August 3, 2021 \\ URL: https://doi.org/10.5430/jnep.v11n12p31
}

\begin{abstract}
Objective: Pediatric patients are considered at risk for Peripheral intravenous catheters (PIVCs) complications more than adults. This study aimed to assess the level of mothers' knowledge of PIVC maintenance, caring, and complications among pediatric patients. It was also aimed to investigate the association between maintenance and complication knowledge of PIVC. Furthermore, demographic factors were investigated to assess associations on mothers' knowledge of PIVC.

Methods: The study adopted a cross-sectional design. This study was performed on a convenience sample of 193 mothers from a tertiary hospital in Jordan in 2020.

Results: Mothers' knowledge regarding complications was higher than their knowledge of maintenance and caring of PIVC. Mothers' knowledge toward caring for PIVC was positively correlated with their knowledge about PIVC complications. Mothers' age and the number of hospital admissions were found to be significantly associated with the level of maintenance and caring knowledge of PIVC but not with complication knowledge of PIVC. The higher the educational level of a mother the less prone she is to complications of PIVC in pediatric patients.

Conclusions: It is recommended that health professionals working in pediatric engage mothers in educational sessions to improve maintenance, care, and to prevent complications of PIVC among pediatric patients.
\end{abstract}

Key Words: Peripheral intravenous catheters, Mothers, Maintenance, Complication, Pediatric

\section{INTRODUCTION}

Peripheral intravenous catheterization (PIVC) is the most common procedure for providing medical treatment such as hydration, medication administration, blood product administration, and nutrition for hospitalized patients. ${ }^{[1]}$ It has been estimated that more than $89 \%$ of hospitalized patients need PIVC and over one billion hospitalized patients during hospitalization have a peripheral intravenous catheter to deliver primary medical treatment. ${ }^{[2]}$ Annually, over 300 million of
PIVC are inserted in The United States. ${ }^{[3]}$ Despite the wide use of PIVC, it is associated with a variety of complications such as phlebitis, infiltration, dislodgment, extravasation, and catheter-associated bloodstream infection. ${ }^{[3]}$

Pediatric patients are considered at risk for PIVCs complications more than adults. For example, Ben Abdelaziz et al., ${ }^{[4]}$ showed that about a quarter of pediatric patients have local complications from PIVC and premature loss of the PIVC before the therapy is completed with a median life

\footnotetext{
* Correspondence: Othman A. Alfuqaha; Email: Othman_alfoqaha@yahoo.com; Address: Department of Nursing, Jordan University Hospital, The University of Jordan, Amman, Jordan.
} 
span of cannula ranging from 29 to 60 hours compared to 96 hours for adult patients. ${ }^{[5]}$ Furthermore, up to $97 \%$ of PIVCs in neonates have these local complications with a median life span from 23 to 40 hours. $^{[4]}$ There has been extensive research regarding the factors of PIVC complication among pediatric patients. ${ }^{[6-8]}$ However, it is widely recognized that the complication factors of PIVC are mainly related to physiological factors such as fragile vein structure and psychological factors such as communication barriers, activity level, little awareness about PIVC importance, and the care dependency on their family. ${ }^{[9]}$ Complications of PIVC are documented in pediatric patients such as phlebitis, infiltration, pain, and local inflammation. It has also been considered a traumatic event for both children and their mothers. ${ }^{[1]}$

The pediatric patient's safety is considered 1 of the 6 domains of quality of care. ${ }^{[10,11]}$ Accordingly, maintaining peripheral intravenous catheters without local complications or premature loss is a way of keeping pediatric patients out of harm. Educational sessions for the pediatric patient caregivers have relatively greater significance in decreased PIVC infiltration and other complications, ${ }^{[12]}$ little attention has been paid to the mothers' knowledge regarding maintenance and complications of PIVC among pediatric patients.

\section{Study aims}

This study aimed to assess the perceived level of mothers' knowledge of PIVC maintenance and complications as primary caregivers. It was also aimed to investigate the association between maintenance and complication knowledge of PIVC. Furthermore, demographic factors such as age, educational level, and numbers of hospital admissions were investigated to assess associations on mothers' knowledge of PIVC. To our knowledge, this is the first study in Jordan that measured mothers' knowledge of handling PIVC and their knowledge of PIVC complications. This study will provide important information about mothers' knowledge of PIVC maintenance and complications in order to enhance mothers' involvement with healthcare providers in providing care for their hospitalized children.

\section{MeTHODS}

\subsection{Design}

The study adopted the cross-sectional design in order to assess the mothers' knowledge regarding PIVC maintenance and PIVC complications. The study adopted the Strengthening the Reporting of Observational Studies in Epidemiology (STROBE) guidelines.

\subsection{Setting}

The study population consisted of mothers admitted into pediatric departments in a major hospital in Amman (the capital of Jordan). Inclusion criteria included: Mothers of inpatient children who stayed in the hospital with their children overnight. Exclusion criteria included: patients who were over 12 years old, patients who were admitted for one day only, and patients who were without PIVC.

\subsection{Participants}

All mothers who were admitted with their child into pediatric departments such as intensive pediatric unit, intermediate pediatric unit, and pediatric floor between the period of June and September in the year 2020 were selected conveniently to participate in this study. After institutional review boards approved this study, we conducted meetings with head nurses and in-charge nurses in the selected areas to explain the study aims. We distributed the envelope that contained the consent form, demographic factors, and study tool to all mothers upon admission time who meet the inclusion criteria. Socio-demographic factors were mothers' age, mothers' educational level, and the number of hospital admissions.

\subsection{Variables}

Mothers' knowledge of PIVC maintenance and mothers' knowledge of PIVC complications are the dependent variables of this study. The independent variables are mothers' age, mothers' educational level, and the number of hospital admissions.

\subsection{Data measurement \\ Mother knowledge scale}

This scale was used to assess the level of maintenance mothers' knowledge of the proper site, dressing clean, hand hygiene, type of duoderm, and clean site of PIVC. Furthermore, this scale was used to assess the level of complications mothers' knowledge and was a sign of redness, swelling, pain, tenderness, and infiltration of PIVC. This scale was adapted from a previously published study of Osti et al. ${ }^{[13]}$ The selfadministered survey was measured on a 5-point Likert scale. For positive items, the scale was as follows: 5 "Always", 4 “Often", 3 "Sometimes", 2 "Rarely", 1 "Never". Negative items were assessed in the reverse order. To assess the overall level, the following standards have been applied as low average score ranged between (1.00-2.33), the moderate average score ranged between (2.34-3.67), and high average score ranged between (3.68-5.00). The study tool consists of 3 parts. The first part was for socio-demographic characteristics which include: mothers' age, educational level, and numbers of hospital admissions. The second part was for measuring mothers' knowledge of maintaining and caring of 
PIVC that consisted of 9 items. The third part consisted of 8 items to measure mother knowledge of PIVC associated complications.

To assess the validity and reliability of the selected scale in the Arabic language; the following criteria were followed: translation process, face validity, construct validity by (factor analysis, Kaiser-Meyer-Olkin (KMO) test for sampling adequacy, and Bartlett's test of sphericity), and Cronbach's alpha was performed as follows: The translation process of mother knowledge survey was examined throughout three steps: forward translation, back translation, and bilingual expert in the related field opinion was assessed. None of the 17-items was removed and hence, they agreed on the final Arabic version. To assess the face validity, a pilot study with 29 mothers was done. We asked them to provide their suggestions regarding context (suitable vs. not suitable), linguistic (appropriate vs. not appropriate), and suitability (stable vs. not stable) of the Arabic version of the survey for the local society. A score more than or equal to 1.5 is considered for all items. ${ }^{[14]}$ A total of $90 \%$ from the pilot study was satisfied regarding the context, linguistic, and suitability of all items. However, we made some amendments in terms of linguistics and local suitability for complication knowledge items.

Factor loadings of the second part (mothers' knowledge of maintaining) were ranged between 0.41 and 0.72 and were ranged between $0.45-0.77$ for the third part (mothers' knowledge of complication). Correlation matrix items were all above 0.40 indicating a strong relationship between items and construct. ${ }^{[15]}$ The KMO test result was 0.92 and 0.89 for maintenance and complication, respectively. This value is considered indicative of sampling adequacy and that factor analysis would yield reliable and distinct factors. ${ }^{[16]}$ Bartlett's test of sphericity: The result shows typically significant and suitable for factor analysis of maintenance survey $\left(\chi^{2}: 2580.60 ; \mathrm{df}: 136 ; p<.001\right)$ and complication survey $\left(\chi^{2}: 1540.45 ;\right.$ df: $\left.128 ; p<.001\right) .{ }^{[17]}$ Finally, to assess the reliability of the mother knowledge scale among participating mothers in this study, the internal consistency reliability was examined. The results revealed Cronbach's Alphas of 0.81 and 0.79 for maintenance and complication items, respectively. These items achieved the required level since the Cronbach's alpha of all constructs is greater than $0.70 .{ }^{[18]}$

\subsection{Statistical methods}

Data were analyzed using the Statistical Package for the Social Sciences version 22 (SPSS). Descriptive statistics were used to assess both types of knowledge of PIVC and other independent variables including mothers' age, mothers' educational level, and the number of hospital admissions. Means, frequencies, standard deviations, and percentages were compared for study variables. Pearson correlation coefficient was used. In addition, a One-way analysis of variance (ANOVA) test was used to find if there is a significant difference between the level of knowledge of PIVC maintenance and the level of knowledge of PIVC complications with other demographic independent variables with an alpha level of (0.05).

\subsection{Ethical approval}

Ethical approval was approved by the institutional review boards of the selected hospital. It has been explained that the mother has the freedom to participate by giving the necessary data. They were informed that they as participants will be coded as numbers to protect their identity and the data did not contain any identifiable information. All subjects were informed that they could contact the corresponding author for any questions and for obtaining the results after completion of the study.

\section{RESULTS}

\subsection{Demographic factors}

The study sample consisted of 230 mothers. Out of the selected sample, 30 mothers were refused to participate indicating a response rate of $80 \%$. The participants were predominately in the age range of 19 to 35 years. In terms of educational level, $33.2 \%$ of participants were pursuing high school or less, $25.4 \%$ were bachelor, and the remaining had diploma degrees and postgraduate degrees. There were 72 mothers admitted more than three times to the hospital, 55 mothers were admitted between two and three times, and the remaining had admitted to the first time (see Table 1).

\subsection{Perceived level and association between mainte- nance and complications of PIVC}

Mothers' knowledge toward complications of PIVC is found to be higher than in maintenance level. Knowledge of the maintenance level of PIVC among mothers is found to be positively associated with knowledge of the complication level. Mothers are aware of the signs/symptoms of complications of PIVC such as redness, swelling, and loss of functioning than caring and maintaining PIVC (see Table 2).

\subsection{Association between maintenance knowledge of PIVC and demographic factors}

The results reveal that there are significant associations between mothers' age and the number of admissions toward their maintenance knowledge of PIVC. On the other hand, results show that there is no significant association between mothers' educational level and their maintenance knowledge of PIVC (see Table 3). 
Table 1. Characteristics of study sample $(\mathrm{N}=193)$

\begin{tabular}{llll}
\hline Variables & Descriptive & Frequency & Percentage (\%) \\
\hline \multirow{2}{*}{ Mothers' Age } & $\leq 18$ years & 17 & 8.8 \\
& 19-35 Years & 103 & 54.4 \\
& $>35$ Years & 73 & 37.8 \\
Educational Level & High School or less & 64 & 33.2 \\
& Diploma degree & 40 & 20.7 \\
& Bachelor's degree & 49 & 25.4 \\
Number of hospital & Postgraduate degree & 40 & 20.7 \\
admissions & First time & 66 & 34.2 \\
& 2-3 times & 55 & 28.5 \\
\hline
\end{tabular}

Table 2. Means, standard deviations, Pearson correlation coefficient, and overall levels for maintenance and complication of PIVC

\begin{tabular}{llllll}
\hline Variable & Mean (\%) & Standard Deviation & Overall Level & $\boldsymbol{r}$ & Sig \\
\hline Maintenance level & 3.38 & 0.58 & Moderate & \multirow{2}{*}{0.21} & $.003^{*}$ \\
Complication level & 3.76 & 0.38 & High & & \\
\hline
\end{tabular}

Note. r: Pearson correlation coefficient. *Sig $\leq .05$.

Table 3. Means, standard deviations, F-distribution, and $p$-level results for mothers' demographic factors and their maintenance knowledge

\begin{tabular}{llll}
\hline Variables & Mean (SD) & F-distribution & Sig \\
\hline Mothers' Age & $3.32(0.83)$ & & $.03 *$ \\
$\leq 18$ years & $3.29(0.57)$ & & \\
$19-35$ Years & $3.52(0.51)$ & & \\
$>35$ Years & & & 1.10 \\
Educational Level & $3.40(0.60)$ & & .35 \\
High School or less & $3.33(0.54)$ & & \\
Diploma degree & $3.29(0.55)$ & & $.02 *$ \\
Bachelor's degree & $3.50(0.62)$ & & 3.69 \\
Postgraduate degree & & \\
Number of hospital admissions & $3.23(0.57)$ & \\
First time & $3.40(0.60)$ & \\
2-3 times & $3.50(0.56)$ & \\
More than 3 Times & & \\
\hline
\end{tabular}

Note. SD: Standard deviation. *Sig $\leq .05$.

\subsection{Association between complication knowledge of PIVC and demographic factors}

There are no significant associations between mothers' age and the number of admissions toward their knowledge about PIVC complications. In contrast, there is a significant association between mothers' educational level and their knowledge about PIVC complications (see Table 4).

Based on their educational level, a post-hoc Scheffe multiple comparison test was performed, and the results showed that there is a statistically significant association between postgraduate degree and other educational levels, where the postgraduate degree is shown to have the highest level of knowledge regarding complications of PIVC (see Table 5).

\section{Discussion}

We found that the level of mother knowledge toward complication is higher than knowledge toward maintenance and care of PIVC. It seems that mothers who are admitted with their children to the hospital are taken into consideration the complications of PIVC regarding signs and symptoms such as pain, redness, tenderness, extravasation, warmth, erythema, and swelling. This result is consistent with previous studies of Mulemba et al. ${ }^{[19]}$ and Suliman et al. ${ }^{[20]}$ Indeed, 
an insertion of PIVC in pediatric patients leading to difficult experiences, pain, and fear is evident. We observed in this study that mothers are aware to choose the proper site of
PIVC, pick the proper type of cannula, select the type of duoderm, wash their hands after using and caring for PIVC.

Table 4. Means, standard deviations, f-distribution, and $p$-level results for mothers' demographic factors and their complication knowledge

\begin{tabular}{|c|c|c|c|}
\hline Variables & Mean (SD) & F-distribution & Sig \\
\hline $\begin{array}{l}\text { Mothers' Age } \\
\leq 18 \text { years } \\
19-35 \text { Years } \\
>35 \text { Years }\end{array}$ & $\begin{array}{l}3.63(0.28) \\
3.78(0.38) \\
3.76(0.37)\end{array}$ & 1.16 & .31 \\
\hline $\begin{array}{l}\text { Educational Level } \\
\text { High School or less } \\
\text { Diploma degree } \\
\text { Bachelor's degree } \\
\text { Postgraduate degree }\end{array}$ & $\begin{array}{l}3.56(0.32) \\
3.75(0.38) \\
3.69(0.28) \\
4.14(0.28)\end{array}$ & 27.15 & $<.001 *$ \\
\hline $\begin{array}{l}\text { Number of hospital admissions } \\
\text { First time } \\
2-3 \text { times } \\
\text { More than } 3 \text { Times }\end{array}$ & $\begin{array}{l}3.71(0.36) \\
3.74(0.43) \\
3.81(0.32)\end{array}$ & 1.42 & .24 \\
\hline
\end{tabular}

Note. SD: Standard deviation. *Sig $\leq .05$.

Table 5. Post-hoc Scheffe multiple comparison test among mothers in terms of their education and complication of PIVC

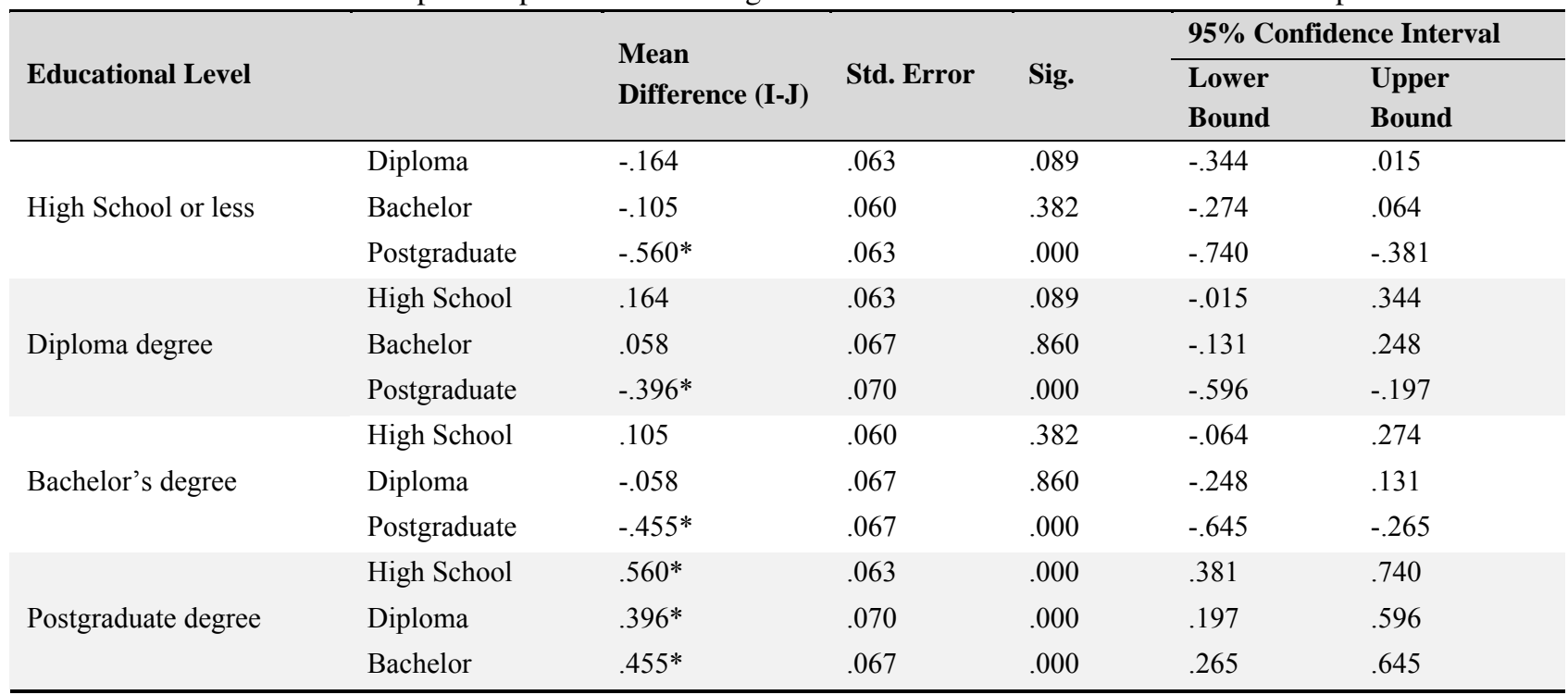

*Sig $\leq .05$.

Mothers' knowledge toward caring for PIVC is positively associated with their knowledge about PIVC complications. This high level of knowledge is strongly related to the perception of IV injections as one of the most feared procedures experienced by hospitalized pediatric patients for both mothers and caregivers. ${ }^{[21]}$ It is widely recognized that mothers are significantly involved in supporting their children psychologically before and during the insertion of PIVC. Mothers' holding and upright positioning proved to be successful in reducing the distress associated with peripheral venous catheter insertion in children. ${ }^{[22]}$

We found that increasing mothers' age and admission number increase mother knowledge about PIVC maintenance. However, this result is in contrast with a study by Al-Ayed ${ }^{[23]}$ which found that neither mothers' age nor their educational levels are affecting mothers' knowledge of their children's 
health issues. The higher the educational level of a mother the less prone she is to complications of PIVC among pediatric patients. Mothers' educational level affects their ability to participate in preventing complications for their hospitalized children. This pattern was also reported by Vasli and Salsali, ${ }^{[24]}$ but disagree with the finding of Naregal et al., ${ }^{[25]}$ who reported that mothers' age and educational level are positively affecting their knowledge of caring for children with Pyrexia. Finally, assess the maintenance and complications knowledge of PIVC among mothers, it would be to prevent pediatric patients from unexpected complications.

\section{Limitations}

Social desirability, convenience sampling by self-reported questionnaires, and ceiling effect might lead to selection and reporting bias. Additionally, we did not measure and consider some important characteristics such as type of mother work, patient acuity status, and geographical area. Finally, the reliability of the tool was established using only internal consistency. Test-retest reliability was not performed. Future studies must take these limitations in their consideration.

\section{Conclusions}

This study shows that mothers' knowledge regarding complications is higher than in the maintenance of PIVC. However, mothers' age and the number of hospital admissions are found to be significantly associated with the level of maintenance and caring knowledge of PIVC but not with the level of complication knowledge of PIVC. Educational level is found to be only associated with the level of complication knowledge. Mothers' knowledge regarding PIVC is important for preventing complications among pediatric patients. We, therefore, recommend that healthcare professionals should enroll the child's parents in educational sessions to improve maintenance, care, and prevent complications of PIVC.

\section{CONFLicts OF InTEREST Disclosure}

The authors report no actual or potential conflicts of interests.

\section{REFERENCES}

[1] Lim EYP, Wong CYW, Kek LK, et al. Improving the Visibility of Intravenous (IV) Site in Pediatric Patients to Reduce IV Site Related Complications - An Evidence-based Utilization Project. Journal of Pediatric Nursing. 2018 Jul-Aug; 41: e39-e45. https: //doi.org/10.1016/j.pedn.2018.04.004

[2] Ray-Barruel G, Xu H, Marsh N, et al. Effectiveness of insertion and maintenance bundles in preventing peripheral intravenous catheterrelated complications and bloodstream infection in hospital patients: A systematic review. Infect Dis Health. 2019 Aug; 24(3): 152-168. https://doi.org/10.1016/j.idh.2019.03.001

[3] Alexandrou E, Ray-Barruel G, Carr PJ, et al. OMG Study Group. Use of Short Peripheral Intravenous Catheters: Characteristics, Management, and Outcomes Worldwide. J Hosp Med. 2018 May 30; 13(5). https://doi.org/10.12788/jhm.3039

[4] Ben Abdelaziz R, Hafsi H, Hajji H, et al. Correction to: Full title: peripheral venous catheter complications in children: predisposing factors in a multicenter prospective cohort study. BMC Pediatr. 2018 Sep 24; 18(1): 307. https : //doi .org/10.1186/s12887-018-1 $281-\mathrm{x}$

[5] Bahl A, Hang B, Brackney A, et al. Standard long IV catheters versus extended dwell catheters: a randomized comparison of ultrasoundguided catheter survival. Am J Emerg Med. 2019; 37: 715-721. https://doi.org/10.1016/j.ajem.2018.07.031

[6] Indarwati F, Mathew S, Munday J, et al. Incidence of peripheral intravenous catheter failure and complications in paediatric patients: Systematic review and meta analysis. Int J Nurs Stud. $2020 \mathrm{Feb}$; 102: 103488. https://doi.org/10.1016/j.ijnurstu. 2019. 103488

[7] Resnick O, Abu Ahmad W, Bancovsky D, et al. Predicting factors for complications in peripheral intravenous catheters in the pediatric population. Acta Paediatr. 2021 May; 110(5): 1639-1644. https://doi.org/10.1111/apa.15687
[8] Tefera M, Letta S, Ararsa A, et al. Incidence and Its Associated Factors of Phlebitis among Pediatric Patients with Peripheral Intravenous Cannula at Hiwot Fana Specialized University Hospital, Harar, Eastern Ethiopia. East African Journal of Health and Biomedical Sciences. 2020 Apr 12; 4(1): 1-2.

[9] Özalp Gerçeker G, Kahraman A, Yardimci F, et al. Infiltration and extravasation in pediatric patients: A prevalence study in a children's hospital. J Vasc Access. 2018 May; 19(3): 266-271. https://doi.org/10.1177/1129729817747532

[10] Franck LS, Ferguson D, Fryda S, et al. The Child and Family Hospital Experience: Is It Influenced by Family Accommodation? Med Care Res Rev. 2015 Aug; 72(4): 419-37. https ://doi .org/10.1 177/1077558715579667

[11] Mueller BU, Neuspiel DR, Fisher ERS. COUNCIL ON QUALITY IMPROVEMENT AND PATIENT SAFETY, COMMITTEE ON HOSPITAL CARE. Principles of Pediatric Patient Safety: Reducing Harm Due to Medical Care. Pediatrics. 2019 Feb; 143(2): e20183649. https://doi.org/10.1542/peds.2018-3649

[12] Park SM, Jeong IS, Kim KL, et al. The Effect of Intravenous Infiltration Management Program for Hospitalized Children. J Pediatr Nurs. 2016 Mar-Apr; 31(2): 172-8. https://doi.org/10.1016/j.pe dn.2015.10.013

[13] Osti C, Khadka M, Wosti D, et al. Knowledge and practice towards care and maintenance of peripheral intravenous cannula among nurses in Chitwan Medical College Teaching Hospital, Nepal. Nurs Open. 2019 Apr 15; 6(3): 1006-1012. https://doi.org/10.1002/no p2. 288

[14] Polit DF, Yang FM. Measurement and the Measurement of Change: A Primer for the Health Professions. Philadelphia: Wolters Kluwer; 2016.

[15] Schreiber JB. Issues and recommendations for exploratory factor analysis and principal component analysis. Res Social Adm Pharm. 2021 May; 17(5): 1004-1011. https://doi.org/10.1016/j.sa pharm.2020.07.027 
[16] Watson JC. Establishing evidence for internal structure using exploratory factor analysis. Measurement and Evaluation in Counseling and Development. 2017 Oct 2; 50(4): 232-8. https://doi.org/ 10.1080/07481756.2017.1336931

[17] Watkins MW. Exploratory factor analysis: A guide to best practice. Journal of Black Psychology. 2018 Apr; 44(3): 219-46. https: //doi.org/10.1177/0095798418771807

[18] Bonett DG, Wright TA. Cronbach's alpha reliability: Interval estimation, hypothesis testing, and sample size planning. Journal of organizational behavior. $2015 \mathrm{Jan} ; 36(1)$ : 3-15. https ://doi .org/ 10.1002/job. 1960

[19] Mulemba T, Bank R, Sabantini M, et al. Improving Peripheral Intravenous Catheter Care for Children with Cancer Receiving Chemotherapy in Malawi. J Pediatr Nurs. 2021 Jan-Feb; 56: 1317. https://doi.org/10.1016/j.pedn.2020.09.019

[20] Suliman M, Saleh W, Al-Shiekh H, et al. The Incidence of Peripheral Intravenous Catheter Phlebitis and Risk Factors among Pediatric Patients. J Pediatr Nurs. 2020 Jan-Feb; 50: 89-93. https: //doi.org/10.1016/j.pedn.2019.11.006
[21] Hwang E, Kim YS, Lee D. Effects of Active Participation and Education of Caregivers on Peripheral Intravenous Injections for Their Child. Int'1 J. Soc. Sci. Stud. 2019; 7: 62-71. https://doi.org/ 10.11114/ijsss.v7i1.3877

[22] Sparks LA, Setlik J, Luhman J. Parental holding and positioning to decrease IV distress in young children: a randomized controlled trial. J Pediatr Nurs. 2007 Dec; 22(6): 440-7. https ://doi .org/10.1 016/j.pedn. 2007.04 .010

[23] Al-Ayed IH. Mothers' knowledge of child health matters: Are we doing enough? J Family Community Med. 2010 Jan; 17(1): 22-8. https://doi.org/10.4103/1319-1683.68785

[24] Vasli P, Salsali M. Parents' participation in taking care of hospitalized children: A concept analysis with hybrid model. Iran J Nurs Midwifery Res. 2014 Mar; 19(2): 139-44.

[25] Naregal P, Mohite VR, Hiremath P, et al. Effectiveness of Health Education on Knowledge of Mothers of Under Five Children Regarding Management of Children Suffering from Pyrexia. Asian Journal of Pharmaceutical Research and Health Care. 2016 Dec 21; 9(1): 22-7. https://doi.org/10.18311/ajprhc/2017/6619 